\title{
Contralateral spontaneous rupture of the esophagus following severe emesis after non-intubated pulmonary wedge resection
}

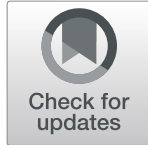

\author{
Lei Liu ${ }^{1,2}$, Wenbin Wu ${ }^{3}$, Longbo Gong ${ }^{3}$ and Miao Zhang ${ }^{3^{*}}$ (D)
}

\begin{abstract}
Background: Non-intubated thoracoscopic lung surgery has been reported to be technically feasible and safe. Spontaneous rupture of the esophagus, also known as Boerhaave's syndrome (BS), is rare after chest surgery.

Case presentation: A 60-year-old female non-smoker underwent non-intubated uniportal thoracoscopic wedge resection for a pulmonary nodule. Ultrasound-guided serratus anterior plane block was utilized for postoperative analgesia. However, the patient suffered from severe emesis, chest pain and dyspnea $6 \mathrm{~h}$ after the surgery. Emergency chest $x$-ray revealed right-sided hydropneumothorax. BS was diagnosed by chest tube drainage and computed tomography. Besides antibiotics and tube feeding, a naso-leakage drainage tube was inserted into the right thorax for pleural evacuation. Finally, the esophagus was healed $40 \mathrm{~d}$ after the conservative treatment.

Conclusions: Perioperative antiemetic therapy is an indispensable item of fast-track surgery. Moreover, BS should be kept in mind when the patients complain of chest distress following emesis after thoracic surgery.

Keywords: Boerhaave's syndrome (BS), Spontaneous ruptures of the esophagus, Three-dimensional CT angiography (3D-CTA), Single port, Uniportal, Video-assisted thoracoscopic surgery (VATS)
\end{abstract}

\section{Background}

Spontaneous rupture of the esophagus, also known as Boerhaave's syndrome (BS), typically occurs after severe emesis as a highly morbid emergency condition [1]. BS accounts for about $15 \%$ of esophageal perforations, and the tears are usually located in lower third of the esophagus [2]. Contrast esophagram and computed tomography $(\mathrm{CT})$ are sufficient for the diagnosis of BS.

Non-intubated video-assisted thoracoscopic surgery (VATS) can be utilized to avoid ventilation-associated adverse effects, which has been reported to be technically feasible and safe [3]. The major complications of non-intubated procedure include intraoperative hypoxia, hypercapnia, and cough.
To our knowledge, the onset of contralateral esophageal rupture after lung resection without lymph node dissection is rare. Herein we presented a case of BS following severe emesis after non-intubated lung surgery. Meanwhile, the current evidence regarding the safety of non-intubated/tubeless thoracic surgery was reviewed briefly.

\section{Case presentation}

The clinical data of the patient were treated anonymously for privacy concern. A 60-year-old previously healthy female non-smoker was admitted because the CT revealed a ground-glass nodule (GGN) about $0.5 \mathrm{~cm}$ in the left upper lobe (Fig. 1a). The serum neuron-

\footnotetext{
* Correspondence: zhangmiaodr@163.com

${ }^{3}$ Department of Surgery, Xuzhou Central Hospital, 199 Jiefang South Road, Xuzhou, China

Full list of author information is available at the end of the article
}

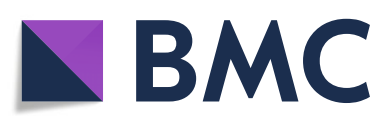

(c) The Author(s). 2020 Open Access This article is licensed under a Creative Commons Attribution 4.0 International License, which permits use, sharing, adaptation, distribution and reproduction in any medium or format, as long as you give appropriate credit to the original author(s) and the source, provide a link to the Creative Commons licence, and indicate if changes were made. The images or other third party material in this article are included in the article's Creative Commons licence, unless indicated otherwise in a credit line to the material. If material is not included in the article's Creative Commons licence and your intended use is not permitted by statutory regulation or exceeds the permitted use, you will need to obtain permission directly from the copyright holder. To view a copy of this licence, visit http://creativecommons.org/licenses/by/4.0/. The Creative Commons Public Domain Dedication waiver (http://creativecommons.org/publicdomain/zero/1.0/) applies to the data made available in this article, unless otherwise stated in a credit line to the data. 


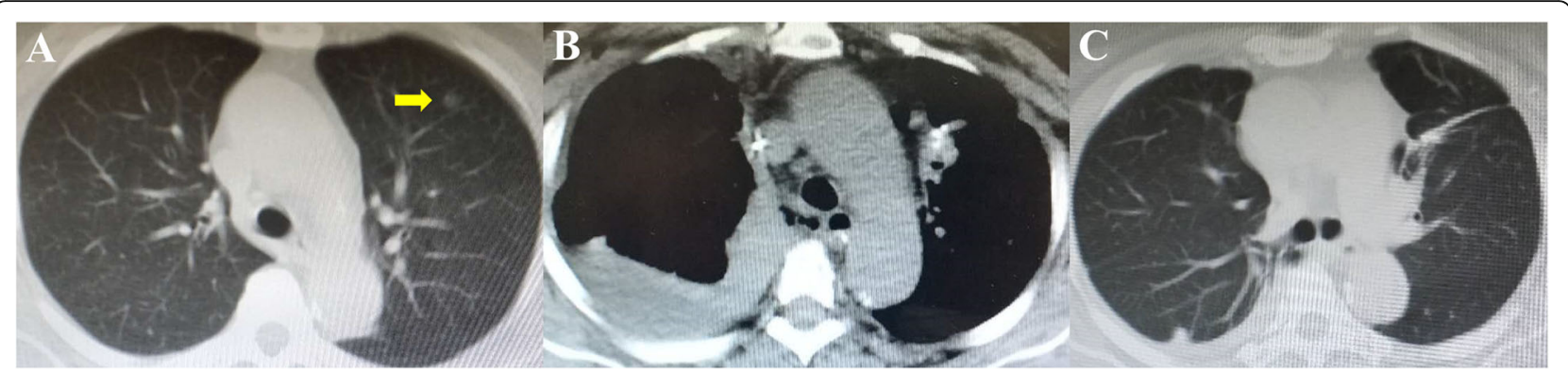

Fig. 1 The computed tomography images. a A nodule in the left upper lobe was indicated by arrow. b The right-sided hydropneumothorax. c The esophagus was healed

specific enolase, cytokeratin-19 fragment, carcinoembryonic antigen, and squamous cell carcinoma were in normal range. After a preoperative workup, the patient was assigned to lung resection. Fast-track protocol was introduced. Preoperative three-dimensional CT angiography (3D-CTA) was established by OsiriX [4]; therefore, invasive labeling of the GGN by microcoil or hook-wire was avoided. Non-intubated uniportal VATS pulmonary wedge resection was performed under internal intercostal nerve block and targeted sedation $[5,6]$. The operation time was $30 \mathrm{~min}$, without obvious blood loss. Mediastinal lymph node sampling wasn't performed because the frozen-section reported atypical adenomatous hyperplasia (AAH). Ultrasound-guided serratus anterior plane block (SAPB) using a bolus of $0.2 \%$ bupivacaine was utilized for postoperative analgesia.

Next-day discharge was scheduled because air leak was not recorded. Postoperative pathological staining of the specimen confirmed the diagnosis of AAH. The patient complained of nausea and emesis about $3 \mathrm{~h}$ after the operation, which was alleviated gradually after intravenous ondansetron (4 mg, once). However, $6 \mathrm{~h}$ after the surgery, she developed sudden tachycardia, tachypnea, dyspnea and hypotension after oral feeding. Emergency CT revealed right-sided hydropneumothorax (Fig. 1b). The turbid, yellow fluid drained from the chest tube further confirmed the diagnosis of BS.

The patient refused a timely surgical intervention. Besides antibiotics and tube feeding, endoscopyguided naso-leakage drainage of the pleural effusion was utilized, which had been reported to be effective to rinse vomica [7]. Finally, the esophagus was healed 40d after the treatment (Fig. 1c). During the 1-year follow up, tumor recurrence or metastasis was not recorded.

\section{Discussion and conclusions}

We identified a patient with BS after severe emesis following minimally invasive lung surgery. Severe emesis is a stressful complication of anesthesia or analgesia. A retrospective study presented 10 patients with esophageal perforation after emesis associated with large volume of food and alcohol intake [8]. Moreover, every perforation was longitudinal tears (about $1-4 \mathrm{~cm}$ ), locating in the left lower-third of the esophagus. Then the authors hypothesized that esophageal perforation probably resulted from emesis through a pathophysiological reaction within the upper digestive tract. Furthermore, they proposed that BS should be defined as post-emetic esophageal perforation. Therefore, we concluded that the present BS was probably secondary to the uncontrolled emesis rather than the surgical procedure itself.

The incidence of post-discharge emesis after ambulatory surgery is approximately 30\% [9]. Chest pain and emesis always suggest the onset of BS, but the patients don't always present with typical clinical features. The major treatment options for BS were conservative, endoscopic and surgical approach; whereas the survival rate of the patients using these treatments was 75, 100 and $81 \%$, respectively [10]. Surgery should be considered especially for those who are admitted within $24 \mathrm{~h}$ of perforation [11]. In addition, endoscopy also plays a role in the treatment of transmural defects [12], although an evidence-based recommendation is still lacking. Besides surgical and endoscopic interventions, naso-esophageal extraluminal drainage has been reported to be effective for the treatment of esophageal leaks and subsequent mediastinal abscess [13].

On the other hand, non-intubated thoracic surgery under minimal sedation with regional anaesthesia is useful to avoid nausea and emesis [14]. However, the evidence supporting non-intubated VATS as the preferred approach for lung surgery is still limited. Previous meta-analyses show that non-intubated procedures could attenuate surgery-related stress responses and decrease postoperative complications compared to intubated surgery $[15,16]$. Moreover, for patients who are considered as high-risk under 
intubated general anesthesia due to their compromised lung function, non-intubated procedure could be considered [17]. A meta-analysis indicates that non-intubated VATS may be a better alternative to intubated surgery [18], although it requires extra vigilance to ensure the safety of the patients [19]. The disadvantages of non-intubated thoracic surgery include cough and poor maneuverability due to the movements of diaphragm and lung [20].

We searched PubMed, Web of Science, Scopus, Embase, Europe PMC, Cochrane Library and Google Scholar for randomized controlled trials (RCTs) up to June 2020 according to the PRISMA Protocol for updated evidence of nonintubated lung surgery. Key words in title or abstract include "non-intubated" or "tubeless" or "awake" and "pulmonary" or "lung" and "surgery". Finally a total of 13 RCTs were obtained (Table 1), which covered 627 patients who underwent nonintubated or tubeless VATS. Among them, 11 (1.8\%) morbidities due to gastrointestinal reactions were recorded. Based on these findings, non-intubated VATS is technically feasible and safe; however, the results should be interpreted with caution due to small samples in the trials and potential publication bias. Well-designed studies are warranted. The registered trials of nonintubated thoracic surgery were listed in Table 2, which might further elucidate the specific indications and contraindications of tubeless thoracic surgery.

In summary, perioperative antiemetic with strict supervision should be considered as an indispensable

Table 1 The reported randomized clinical trials regarding non-intubated thoracoscopic lung surgery

\begin{tabular}{|c|c|c|c|c|c|c|c|}
\hline $\begin{array}{l}\text { First } \\
\text { author, } \\
\text { year }\end{array}$ & Sample & $\begin{array}{l}\text { Age, } \\
\text { year }\end{array}$ & $\begin{array}{l}\text { Anaesthesia } \\
\text { method }\end{array}$ & Surgical procedure & $\begin{array}{l}\text { Conversion } \\
\text { to } \\
\text { intubation }\end{array}$ & $\begin{array}{l}\text { Postoperative } \\
\text { analgesia }\end{array}$ & $\begin{array}{l}\text { Morbidity due to } \\
\text { gastrointestinal } \\
\text { reactions }\end{array}$ \\
\hline $\begin{array}{l}\text { Pompeo, } \\
2004[21]\end{array}$ & 30 & $\begin{array}{l}60 \\
(45- \\
68)\end{array}$ & TEA at T4-T5 & Pulmonary nodule resection & $4(13.3 \%)$ & TEA & $N R$ \\
\hline $\begin{array}{l}\text { Pompeo, } \\
2007[22]\end{array}$ & 21 & $\begin{array}{l}28 \pm \\
14\end{array}$ & $\begin{array}{l}\text { Locoregional } \\
\text { anaesthesia }\end{array}$ & Bullectomy & 0 & TEA & $1(4.8 \%)$ \\
\hline $\begin{array}{l}\text { Vanni, } \\
2010[23]\end{array}$ & 25 & $\begin{array}{l}57 \\
(51- \\
62)\end{array}$ & TEA & $N R$ & 0 & $\mathrm{PCIA}$ & 0 \\
\hline $\begin{array}{l}\text { Tacconi, } \\
2010[24]\end{array}$ & 11 & $\begin{array}{l}48 \\
(43- \\
55)\end{array}$ & TEA & $\begin{array}{l}\text { Lung nodule resection, bullectomy, pleura- } \\
\text { lung biopsy }\end{array}$ & 0 & $\mathrm{PCIA}$ & 0 \\
\hline $\begin{array}{l}\text { Pompeo, } \\
2011[25]\end{array}$ & 32 & $64 \pm 9$ & TEA at T4-5 & Lung volume reduction & $2(6.3 \%)$ & $N R$ & 0 \\
\hline $\begin{array}{l}\text { Pompeo, } \\
2013[26]\end{array}$ & 20 & $\begin{array}{l}67 \pm \\
12\end{array}$ & TEA at T4 & Pleurodesis & 0 & $N R$ & 0 \\
\hline $\begin{array}{l}\text { Cai, } 2013 \\
{[27]}\end{array}$ & 30 & $\begin{array}{l}23.5 \pm \\
10.6\end{array}$ & $\begin{array}{l}\text { Laryngeal mask } \\
\text { anesthesia }\end{array}$ & Bullectomy & 0 & $\mathrm{PCIA}$ & $3(10.0 \%)$ \\
\hline $\begin{array}{l}\text { Wang, } \\
2014[28]\end{array}$ & 50 & $\begin{array}{l}43.2 \pm \\
14.7\end{array}$ & $\begin{array}{l}\text { General anesthesia; } \\
\text { laryngeal mask }\end{array}$ & $\begin{array}{l}\text { Bullectomy, lobectomy, biopsy, } \\
\text { mediastinal mass excision }\end{array}$ & 0 & $N R$ & 0 \\
\hline $\begin{array}{l}\text { Liu, } 2015 \\
{[29]}\end{array}$ & 167 & $N R$ & TEA & Wedge resection, lobectomy & 0 & $N R$ & $4(2.4 \%)$ \\
\hline $\begin{array}{l}\text { Chen, } \\
2016[30]\end{array}$ & 85 & $\begin{array}{l}23.3 \pm \\
6.8\end{array}$ & $\begin{array}{l}\text { Intravenous } \\
\text { anesthesia }\end{array}$ & Sympathectomy & 0 & $N R$ & 0 \\
\hline $\begin{array}{l}\text { Mao, } 2018 \\
{[31]}\end{array}$ & 30 & $\begin{array}{l}21 \pm \\
3.2\end{array}$ & $\begin{array}{l}\text { General anesthesia + } \\
\text { laryngeal mask }\end{array}$ & NUSS procedure & 0 & $\mathrm{PCIA}$ & $3(10.0 \%)$ \\
\hline $\begin{array}{l}\text { Hwang, } \\
2018 \text { [32] }\end{array}$ & 21 & $\begin{array}{l}17 \\
(17- \\
45)\end{array}$ & Sedation anesthesia & Bullectomy & 0 & Local analgesia & 0 \\
\hline \multirow[t]{3}{*}{$\begin{array}{l}\text { Mogahed, } \\
2019 \text { [33] }\end{array}$} & 35 & $\begin{array}{l}42.9 \pm \\
9.6\end{array}$ & General anaesthesia & \multirow{3}{*}{$\begin{array}{l}\text { Lung resections, excision/biopsy of } \\
\text { mediastinal mass, foreign body extraction } \\
\text { and pericardial window. }\end{array}$} & \multirow[t]{3}{*}{0} & \multirow[t]{3}{*}{$\begin{array}{l}\text { Intramuscular } \\
\text { ketoprofen }\end{array}$} & \multirow[t]{3}{*}{ NR } \\
\hline & 35 & $\begin{array}{l}43.5 \pm \\
10.5\end{array}$ & $\begin{array}{l}\text { General anaesthesia } \\
+ \text { TEA }\end{array}$ & & & & \\
\hline & 35 & $\begin{array}{l}44.0 \pm \\
9.3\end{array}$ & $\begin{array}{l}\text { General anaesthesia } \\
+ \text { intercostal block } \\
\text { infiltration }\end{array}$ & & & & \\
\hline
\end{tabular}


Table $\mathbf{2}$ The registered trials of non-intubated or tubeless thoracoscopic lung surgery

\begin{tabular}{|c|c|c|c|c|c|c|c|}
\hline $\begin{array}{l}\text { Registration } \\
\text { identifier }\end{array}$ & Year & Disease & Anaesthesia method & $\begin{array}{l}\text { Estimated } \\
\text { enrollment }\end{array}$ & Major outcomes & Status & Country \\
\hline NCT00566839 & 2007 & Emphysema & TEA & 60 & $\begin{array}{l}\text { Mortality, FEV1, } \\
\text { dyspnea index }\end{array}$ & Completed & Italy \\
\hline NCT01469728 & 2011 & NR & TEA & 40 & $\begin{array}{l}\text { Grade of medical } \\
\text { care }\end{array}$ & Completed & Italy \\
\hline NCT01677442 & 2011 & NR & TEA at the T5/T6 & 500 & Recovery time & Unknown & China \\
\hline NCT01533233 & 2012 & Lung cancer & NR & 100 & $\begin{array}{l}\text { Complication and } \\
\text { morbidity }\end{array}$ & Unknown & China \\
\hline NCT02109510 & 2014 & Pneumothorax & $\begin{array}{l}\text { Sedation anesthesia + intercostal nerve } \\
\text { block }\end{array}$ & 40 & $\begin{array}{l}\text { Postoperative } \\
\text { discomforts }\end{array}$ & Completed & Korea \\
\hline NCT02123173 & 2014 & Lung neoplasms & NR (one lung ventilation) & 71 & Cardiac output & Completed & China \\
\hline NCT02393664 & 2015 & Lung neoplasms & $\begin{array}{l}\text { General anesthesia + intercostal/vagal } \\
\text { blocks }\end{array}$ & 300 & Quality of recovery & Unknown & China \\
\hline NCT02817048 & 2016 & $\begin{array}{l}\text { Solitary lung } \\
\text { nodule }\end{array}$ & NR (Tubeless) & 100 & $\begin{array}{l}\text { Postoperative } \\
\text { hospital stay }\end{array}$ & $\begin{array}{l}\text { Not yet } \\
\text { recruiting }\end{array}$ & China \\
\hline NCT03275428 & 2017 & Lung nodule & Intravenous sedation & 40 & $\begin{array}{l}\text { Arterial oxygen } \\
\text { pressure }\end{array}$ & Unknown & China \\
\hline NCT03083080 & 2017 & NR & Intercostal nerve plane block & 30 & $\begin{array}{l}\text { Pain, time to lose } \\
\text { skin sensation }\end{array}$ & Unknown & China \\
\hline NCT03086213 & 2017 & NR & Paravertebral/intercostal nerve block & 48 & $\begin{array}{l}\text { The change of } \\
\text { stress response } \\
\text { markers }\end{array}$ & Unknown & China \\
\hline NCT03016858 & 2017 & Bulla & Intravenous anesthesia & 320 & Complications & Recruiting & China \\
\hline NCT03137576 & 2017 & Lung neoplasms & $\begin{array}{l}\text { Erector spinae plane block/paravertebral } \\
\text { block and sedation }\end{array}$ & 172 & $\begin{array}{l}\text { Percentage of } \\
\text { sedation escalation }\end{array}$ & Recruiting & Italy \\
\hline $\begin{array}{l}\text { ChiCTR-INR- } \\
17012747\end{array}$ & 2017 & Thoracic diseases & General anesthesia & 30 & $\begin{array}{l}\text { Length of hospital } \\
\text { stay }\end{array}$ & Recruiting & China \\
\hline $\begin{array}{l}\text { ChiCTR-IPR- } \\
17013325\end{array}$ & 2017 & Lung nodule & Intravenous anesthesia & 120 & $\begin{array}{l}\text { CD3+, CD8+, CD4+, } \\
\text { CD19+, NK cell } \\
\text { concentration }\end{array}$ & $\begin{array}{l}\text { Not yet } \\
\text { recruiting }\end{array}$ & China \\
\hline NCT03711461 & 2018 & NR & NR & 32 & $\begin{array}{l}\text { Impedance changes } \\
\text { (swallowing) }\end{array}$ & Recruiting & China \\
\hline NCT03432637 & 2018 & Lung cancer & Spontaneous ventilating anesthesia & 450 & $\begin{array}{l}\text { Hypoxemia or } \\
\text { hypercapnia }\end{array}$ & Recruiting & China \\
\hline NCT03471884 & 2018 & Lung cancer & General anesthesia & 82 & Lung function & Recruiting & China \\
\hline NCT03469323 & 2018 & NR & NR (one-lung spontaneous breathing) & 30 & $\begin{array}{l}\text { Quality of lung } \\
\text { collapse }\end{array}$ & Recruiting & China \\
\hline ChiCTR1800018198 & 2018 & NR & Paravertebral nerve block + laryngeal mask & 110 & $\begin{array}{l}\text { Glottal injury, sore } \\
\text { throat }\end{array}$ & Recruiting & China \\
\hline NCT03653494 & 2018 & NR & $\begin{array}{l}\text { General anesthesia + paravertebral block }+ \\
\text { surface spray anesthesia + vagus block with } \\
\text { or without phrenic block }\end{array}$ & 80 & $\begin{array}{l}\text { Anesthetic drugs } \\
\text { needed }\end{array}$ & $\begin{array}{l}\text { Enrolling } \\
\text { by } \\
\text { invitation }\end{array}$ & China \\
\hline ChiCTR1800018204 & 2018 & NR & $\begin{array}{l}\text { Serratus anterior plane/erector spinae } \\
\text { plane/paravertebral block }\end{array}$ & 90 & Nerve block time & $\begin{array}{l}\text { Not yet } \\
\text { recruiting }\end{array}$ & China \\
\hline ChiCTR1800017854 & 2018 & $\begin{array}{l}\text { T1a }(<2 \mathrm{~cm}) \\
\text { peripheral lung } \\
\text { adenocarcinoma }\end{array}$ & NR (Tubeless) & 200 & Complications & $\begin{array}{l}\text { Not yet } \\
\text { recruiting }\end{array}$ & China \\
\hline NCT03874403 & 2019 & NR & Intercostal nerve block & 60 & $\begin{array}{l}\text { The density spectral } \\
\text { array }\end{array}$ & Recruiting & China \\
\hline NCT04057586 & 2019 & NR & NR (one lung ventilation) & 240 & $\begin{array}{l}\text { Intraoperative } \\
\text { cerebral } \\
\text { oxygenation }\end{array}$ & Recruiting & China \\
\hline ChiCTR1900027350 & 2019 & Lung cancer & $\begin{array}{l}\text { Intercostal/paravertebral nerve block + } \\
\text { general anesthesia using laryngeal mask }\end{array}$ & 80 & $\begin{array}{l}\text { Hemodynamics, } \\
\text { general anesthetic }\end{array}$ & Recruiting & China \\
\hline
\end{tabular}


Table 2 The registered trials of non-intubated or tubeless thoracoscopic lung surgery (Continued)

\begin{tabular}{|c|c|c|c|c|c|c|c|}
\hline $\begin{array}{l}\text { Registration } \\
\text { identifier }\end{array}$ & Year & Disease & Anaesthesia method & $\begin{array}{l}\text { Estimated } \\
\text { enrollment }\end{array}$ & Major outcomes & Status & Country \\
\hline & & & & & dose, recovery time & & \\
\hline ChiCTR1900022020 & 2019 & Thoracic disease & General anesthesia & 120 & $\begin{array}{l}\text { Glottal injury } \\
\text { incidence, lung } \\
\text { collapse score }\end{array}$ & Recruiting & China \\
\hline NCT03958162 & 2019 & $\begin{array}{l}\text { Interstitial lung } \\
\text { disease }\end{array}$ & NR (tubeless) & 60 & $\begin{array}{l}\text { Diagnostic yield } \\
\text { after biopsy }\end{array}$ & $\begin{array}{l}\text { Not yet } \\
\text { recruiting }\end{array}$ & China \\
\hline NCT03902470 & 2019 & Lung cancer & TEA & 30 & Recovery time & $\begin{array}{l}\text { Not yet } \\
\text { recruiting }\end{array}$ & Egypt \\
\hline
\end{tabular}

TEA thoracic epidural anaesthesia; FEV1 Forced expiratory volume in one second; NR not reported

item of fast-track thoracic surgery. Moreover, the occurrence of BS and a timely intervention should be kept in mind when the patients report chest distress after severe emesis following lung surgery.

\section{Abbreviations}

CT: Computed tomography; BS: Boerhaave's syndrome; VATS: Video-assisted thoracoscopic surgery; AAH: Atypical adenomatous hyperplasia; SAPB: Serratus anterior plane block; RCTs: Randomized controlled trials

\section{Acknowledgements}

Not applicable.

\section{Authors' contributions}

$\mathrm{MZ}$ performed the surgery and wrote this paper. $\mathrm{LL}$ contributed to the preparation of the figures and tables. All authors contributed to preparation of the paper and to the perioperative treatment of the patient. All authors approved the final manuscript.

\section{Funding}

Not applicable.

\section{Availability of data and materials}

The data used in this report are available from the corresponding author on reasonable request.

\section{Ethics approval and consent to participate}

This report was approved by the Institutional Review Board of Xuzhou Central Hospital.

\section{Consent for publication}

Written informed consent was obtained from the patient for publication of this report and any accompanying images.

\section{Competing interests}

The authors declare that they have no competing interests.

\section{Author details}

${ }^{1}$ Institute of Digestive Disease, China Three Gorges University, Yichang, China. 2Department of Gastroenterology, Yichang Central People's Hospital, Yichang, China. ${ }^{3}$ Department of Surgery, Xuzhou Central Hospital, 199 Jiefang South Road, Xuzhou, China.

Received: 22 June 2020 Accepted: 21 September 2020

Published online: 01 October 2020

\section{References}

1. Shiber JR, Fontane E, Ra JH, Kerwin AJ. Hydropneumothorax due to esophageal rupture. J Emerg Med. 2017;52:856-8.

2. Chirica M, Kelly MD, Siboni S, et al. Esophageal emergencies: WSES guidelines. World J Emerg Surg. 2019;14:26.
3. He J, Liu J, Zhu C, et al. Expert consensus on tubeless video-assisted thoracoscopic surgery (Guangzhou). J Thorac Dis. 2019;11:4101-8.

4. Zhang M, Liu D, Wu W, Zhang H, Mao N. Preoperative 3D-CT bronchography and angiography facilitates single-direction uniportal thoracoscopic anatomic lobectomy. Ann Transl Med. 2019;7:526.

5. Longo F, Piliego C, Tomaselli E, Martuscelli M, Agro FE. Erector spinae plane block allows non-intubated vats-wedge resection. J Clin Anesth. 2020:60:89-90.

6. Hung MH, Hsu HH, Chan KC, et al. Non-intubated thoracoscopic surgery using internal intercostal nerve block, vagal block and targeted sedation. Eur J Cardiothorac Surg. 2014;46:620-5.

7. Zhang $Y$, Zhang $Y X, H u$ JW, et al. Endoscopic naso-leakage drainage: a safe and effective method for the management of intrathoracic anastomotic leakage after esophagectomy. J Thorac Dis. 2017;9:3052-61.

8. Kuwano $H$, Yokobori $T$, Kumakura $Y$, et al. Pathophysiology of vomiting and esophageal perforation in Boerhaave's syndrome. Dig Dis Sci. 2020. https:// doi.org/10.1007/s10620-019-06028-0 Online ahead of print.

9. Mattila K, Toivonen J, Janhunen L, Rosenberg PH, Hynynen M. Postdischarge symptoms after ambulatory surgery: first-week incidence, intensity, and risk factors. Anesth Analg. 2005;101:1643-50.

10. de Schipper JP, Pull ter Gunne AF, Oostvogel HJ, et al. Spontaneous rupture of the oesophagus: Boerhaave's syndrome in 2008. Literature review and treatment algorithm. Dig Surg. 2009;26:1-6.

11. Nakano T. Surgical techniques of spontaneous esophageal rupture. Kyobu Geka. 2019;72:878-85.

12. Bemelman WA, Baron TH. Endoscopic Management of Transmural Defects, including leaks, perforations, and fistulae. Gastroenterology. 2018;154:193846 e1931.

13. Shuto K, Kono T, Akutsu Y, et al. Naso-esophageal extraluminal drainage for postoperative anastomotic leak after thoracic esophagectomy for patients with esophageal cancer. Dis Esophagus. 2017;30:1-9.

14. Irons JF, Martinez G. Anaesthetic considerations for non-intubated thoracic surgery. J Vis Surg. 2016;2:61

15. Yu MG, Jing R, Mo YJ, et al. Non-intubated anesthesia in patients undergoing video-assisted thoracoscopic surgery: A systematic review and meta-analysis. PLoS One. 2019;14:e0224737.

16. Wen $Y$, Liang $H$, Qiu G, et al. Non-intubated spontaneous ventilation in video-assisted thoracoscopic surgery: a meta-analysis. Eur J Cardiothorac Surg. 2020;57:428-37.

17. Wang ML, Hung MH, Hsu HH, Chan KC, Cheng YJ, Chen JS. Non-intubated thoracoscopic surgery for lung cancer in patients with impaired pulmonary function. Ann Transl Med. 2019;7:40.

18. Zhang K, Chen HG, Wu WB, et al. Non-intubated video-assisted thoracoscopic surgery vs. intubated video-assisted thoracoscopic surgery for thoracic disease: a systematic review and meta-analysis of 1,684 cases. J Thorac Dis. 2019:11:3556-68.

19. Shi $Y, Y u H$, Huang $L$, et al. Postoperative pulmonary complications and hospital stay after lung resection surgery: A meta-analysis comparing nonintubated and intubated anesthesia. Medicine (Baltimore). 2018;97: e10596.

20. Pompeo E, Sorge R, Akopov A, et al. Non-intubated thoracic surgery-a survey from the European Society of Thoracic Surgeons. Ann Transl Med. 2015;3:37. 
21. Pompeo E, Mineo D, Rogliani P, Sabato AF, Mineo TC. Feasibility and results of awake thoracoscopic resection of solitary pulmonary nodules. Ann Thorac Surg. 2004;78:1761-8.

22. Pompeo $E$, Tacconi $F$, Mineo $D$, Mineo TC. The role of awake video-assisted thoracoscopic surgery in spontaneous pneumothorax. J Thorac Cardiovasc Surg. 2007;133:786-90.

23. Vanni G, Tacconi F, Sellitri F, Ambrogi V, Mineo TC, Pompeo E. Impact of awake videothoracoscopic surgery on postoperative lymphocyte responses. Ann Thorac Surg. 2010;90:973-8.

24. Tacconi F, Pompeo E, Sellitri F, Mineo TC. Surgical stress hormones response is reduced after awake videothoracoscopy. Interact Cardiovasc Thorac Surg. 2010;10:666-71.

25. Pompeo E, Rogliani P, Tacconi F, et al. Randomized comparison of awake nonresectional versus nonawake resectional lung volume reduction surgery. J Thorac Cardiovasc Surg. 2012;143:47-54 54 e41.

26. Pompeo E. Dauri M and awake thoracic surgery research group. Is there any benefit in using awake anesthesia with thoracic epidural in thoracoscopic talc pleurodesis? J Thorac Cardiovasc Surg. 2013;146:495-7 e491.

27. Cai $K$, Wang $X$, Ye J, et al. Laryngeal mask anesthesia in video-assisted thoracoscopic surgery for pulmonary bulla: comparison with intubation anesthesia. Nan Fang Yi Ke Da Xue Xue Bao. 2013;33:756-60.

28. Wang S, Zhang J, Cheng H, Yin J, Liu X. A clinical evaluation of the ProSeal laryngeal mask airway with a Coopdech bronchial blocker for one-lung ventilation in adults. J Cardiothorac Vasc Anesth. 2014;28:900-3.

29. Liu J, Cui F, Li S, et al. Nonintubated video-assisted thoracoscopic surgery under epidural anesthesia compared with conventional anesthetic option: a randomized control study. Surg Innov. 2015;22:123-30.

30. Chen J, Du Q, Lin M, et al. Transareolar single-port Needlescopic thoracic Sympathectomy under intravenous anesthesia without intubation: a randomized controlled trial. J Laparoendosc Adv Surg Tech A. 2016;26:958-64.

31. Mao S, Du X, Ma J, Zhang G, Cui J. A comparison between laryngeal mask airway and endotracheal intubation for anaesthesia in adult patients undergoing NUSS procedure. J Thorac Dis. 2018;10:3216-24.

32. Hwang J, Shin JS, Son JH, Min TJ. Non-intubated thoracoscopic bullectomy under sedation is safe and comfortable in the perioperative period. J Thorac Dis. 2018;10:1703-10.

33. Mogahed MM, Elkahwagy MS. Paravertebral block versus intercostal nerve block in non-intubated Uniportal video-assisted thoracoscopic surgery: a randomised controlled trial. Heart Lung Circ. 2020;29:800-7.

\section{Publisher's Note}

Springer Nature remains neutral with regard to jurisdictional claims in published maps and institutional affiliations.

Ready to submit your research? Choose BMC and benefit from:

- fast, convenient online submission

- thorough peer review by experienced researchers in your field

- rapid publication on acceptance

- support for research data, including large and complex data types

- gold Open Access which fosters wider collaboration and increased citations

- maximum visibility for your research: over $100 \mathrm{M}$ website views per year

At $\mathrm{BMC}$, research is always in progress.

Learn more biomedcentral.com/submissions 